\section{Roadmap to adversity}

\section{By Michael J. Haas, Senior Writer}

Novartis AG researchers have generated a statistical map that correlates chemical features of approved drugs with adverse reactions to the molecules. ${ }^{1}$ The pharma is already using the map to help guide preclinical and clinical research and hopes it will be able to elucidate some of the mechanisms that underlie off-target effects.

Because of the reams of data on drug structure and side effects, previous efforts by academics and companies to link the two have focused on a single drug at a time. The Novartis team overcame this onesy-twosy approach with brute force-they manually compiled all the data into a format amenable to chemical informatics analysis. The result is the ability to look for correlations across the entire body of available data, according to team coleader Jeremy Jenkins.

"There are many researchers out there who don't have this kind of information because of the hurdles involved in manual assembly," said Jenkins, a research investigator at the Novartis Institute for Biomedical Research. "If everyone had this kind of data, this area of research would explode with activity."

Indeed, Novartis has made publicly available the raw data accompanying its Journal of Medicinal Chemistry paper.

The JMC map built on a 2007 report in which the same Novartis team described links between an adverse reaction and chemical features shared by drugs that caused it. ${ }^{2}$ In the new article, the group went further and linked multiple adverse reactions to a given structure, even when those reactions occurred in different organs or systems of the body, said team coleader Josef Scheiber, who is also a research investigator at Novartis Institute for Biomedical Research.

To construct the map, the team compiled data on 4,210 types of adverse reactions reported in the PharmaPendium database of 1,842 marketed drugs (https://www.pharmapendium.com/). They then developed a mathematical representation of each drug's structure.

Next, the team looked for statistical correlations between every possible pair of adverse reactions-a total of almost 9 million-and any 1 of about 10,000 chemical features represented in the drug structures. The result was 30,000 significant structure-reaction correlations involving 1,501 distinct adverse reactions.

The team mapped the results in $2 \mathrm{D}$, using a dot to represent each adverse reaction. Lines between dots represented adverse reactions that correlated to one or more shared chemical features. The closer the linked dots were on the map, the tighter the structure-based correlation.

The 2D map allowed "visualization of adverse drug reactions that are related in chemical space, even if they are not linked to the same biology," Scheiber said.

Additionally, the team color-coded each dot according to the organ or system in which the adverse reaction occurred. "Now you can see clusters of information and novel or unexpected connections and relationships" aided by the organ- and system-specific color-coding, said Jenkins.

Both researchers cautioned against assuming that there was a simple one-to-one correspondence between dot connections and structures. "When reading the map, it is important to remember that each correlation has many underlying substructures-maybe 100 each," Scheiber told SciBX.

"Novartis should be congratulated for their innovative approach in this important area of research," said David Allan, SVP of GlaxoSmithKline plc's Respiratory Centre of Excellence for External Drug Discovery. "Their novel analysis and preliminary conclusions are intriguing and raise many opportunities for further work and discussion in this area," especially because the raw data have been made available.

Allan told SciBX that GSK "will be examining the methodology in depth to see how it can complement our own approaches to understanding and minimizing adverse drug reactions in the future."

Last year, GSK launched Molecular Clinical Safety Intelligence (MCSI), a software system that the company uses to screen discoverystage compounds for potential adverse reactions. GSK developed MCSI with Phase Forward Inc., which provides integrated data management solutions for clinical trials and drug safety applications.

\section{Map quest}

Unlike GSK's software, Novartis is not yet using its map in discovery. Instead, the current focus is using the map to help drive preclinical and clinical research.

For example, Jenkins said Novartis has already used its map to understand the side effects seen with an undisclosed compound in clinical development on the basis of structure.

He said the team is also combing the map for side effects that Novartis might want to add to its routine safety screens and profiles, according to the chemical structure of a compound in development.

Although the map could drive drug design by predicting likely adverse reactions for compounds at the discovery stage, both Scheiber and Jenkins said the map has more utility in preclinical and clinical development.

"The whole analysis is based on the structures of marketed drugs" whose chemical features contribute to desirable properties as well as adverse reactions, Scheiber said. "If you start by engineering out all 
of the structures with predicted side effects, you might also weed out structural features that give the drug other desirable features, such as bioavailability or activity."

Indeed, Jenkins noted that "it would probably be a mistake to apply this map too early in the drug discovery process."

The team is continuing to expand the map by linking the adverse reaction-structure correlations to protein targets-including those that are not addressed by approved drugs—and related biological pathways.

"We have thousands of examples from other statistical models to predict likely targets of a particular chemical feature, and thus link structure to mechanism," said Jenkins. By combining that pharmacological information with the 2D map "you get a sneak preview of potential adverse drug reactions," he said.

Scheiber thinks this expanded map will find use in discovery research by enabling the design of assays to screen new compounds for predicted toxicities.

He said the team expects to publish a paper about the protein target dimension this year.

Jenkins said Novartis has not patented or licensed the findings reported in JMC. “The company is not interested in patenting ideas in this 'precompetitive space,"' he said.

Novartis also participates in FDA's Critical Path Initiative as a member of the Predictive Safety Testing Consortium (PSTC), which investigates potential toxicity biomarkers under the aegis of The Critical Path Institute, a not-for-profit organization.

Scheiber said that his team's work is not connected to the pharma's involvement in the PSTC.

Haas, M.J. SciBX 2(19); doi:10.1038/scibx.2009.776

Published online May 14, 2009

\section{REFERENCES}

1. Scheiber, J. et al. J. Med. Chem.; published online April 20, 2009; doi:10.1021/jm801546k

Contact: Josef Scheiber, Novartis Institutes for Biomedical Research, Cambridge, Mass.

e-mail: mail@josef-scheiber.de

2. Bender, A. et al. ChemMedChem 2, 861-873 (2007)

COMPANIES AND INSTITUTIONS MENTIONED

The Critical Path Institute, Tucson, Ariz.

Food and Drug Administration, Silver Spring, Md.

GlaxoSmithKline plc (LSE:GSK; NYSE:GSK), London, U.K.

Novartis AG (NYSE:NVS; SIX:NOVN), Basel, Switzerland

Phase Forward Inc. (NASDAQ:PFWD), Waltham, Mass. 